Carvalho, GAO, Almeida, RR, Câmara, JVF, Ribeiro, AOP \& Peirote, JJA. (2020). Stratification of resin composed with use of silicone barrier: literature review. Research, Society and Development, 9(7):1-16, e246974114.

\title{
Estratificação da resina composta com o uso de barreira de silicone: revisão de literatura
} Stratification of resin composed with use of silicone barrier: literature review

Estratificación de resina compuesta con el uso de barrera de silicona: revisión de literatura

Recebido: 30/04/2020 | Revisado: 01/05/2020 | Aceito: 04/05/2020 | Publicado: 10/05/2020

Guereth Alexsanderson Oliveira Carvalho ORCID: https://orcid.org/0000-0003-3286-2943

Universidade Federal do Piauí, Brasil E-mail: guerethcarvalho@gmail.com

Rubiana Romão de Almeida

ORCID: https://orcid.org/0000-0001-5939-7645 Instituto Nacional de Ensino e Pós-Graduação Gervásio, Brasil E-mail: rubiana-almeida@hotmail.com

João Victor Frazão Câmara ORCID: https://orcid.org/0000-0002-9687-4401

Universidade de São Paulo, Brasil

E-mail: jvfrazao92@hotmail.com

Amanda de Oliveira Pinto Ribeiro

ORCID: https://orcid.org/0000-0003-4705-6848 Universidade Estadual Paulista Júlio de Mesquita Filho, Brasil E-mail: amandaribeiro11.2@gmail.com Josué Junior Araujo Pierote ORCID: https://orcid.org/0000-0003-0585-1405

Universidade de Santo Amaro, Brasil E-mail: josuepierote@ hotmail.com

\section{Resumo}

A odontologia estética encontra-se em contínuo avanço devido aos procedimentos adesivos e ao desenvolvimento de materiais restauradores que buscam a melhora das propriedades mecânicas e estéticas para reprodução das características naturais das estruturas dentais. Este estudo tem como objetivo destacar as vantagens clínicas do seu uso, bem como sua aplicação 
clínica através de uma revisão de literatura. Foram utilizadas as palavras-chave "restauração estética", "estratificação" e "barreira de silicone" nas bases de dados PubMed e BVS. Foram escolhidas as referências que tratavam dos assuntos relacionados ao tema proposto, publicadas entre 1993 e 2020 . As resinas compostas atuais possibilitam restaurar a forma e a função com as características de cor, translucidez e opacidade semelhantes às dos dentes naturais, sendo indicadas para a recuperação da harmonia do sorriso anterior. $O$ uso da barreira de silicone para a realização das reabilitações estéticas anteriores facilita o processo de estratificação, e a recuperação estética do sorriso como pode ser observado através desta revisão de literatura.

Palavras-chave: Resinas compostas; Silicones; Estética dentária.

\begin{abstract}
Aesthetic dentistry is constantly advancing due to adhesive procedures and the development of restorative materials that seek to improve mechanical and aesthetic properties to reproduce the natural characteristics of dental structures. This study aims to highlight the clinical advantages of its use, as well as its clinical application through a literature review. The keywords "composite resins", "silicones" and "esthetics, dental" were used in the PubMed and BVS databases. The references dealing with the subjects related to the proposed theme were chosen, published between 1993 and 2020. The current composite resins make it possible to restore the shape and function with the characteristics of color, translucency and opacity similar to those of natural teeth, being indicated for recovery the harmony of the previous smile. The use of the silicone barrier to perform previous aesthetic rehabilitation facilitates the stratification process, and the aesthetic recovery of the smile as can be seen through this literature review.
\end{abstract}

Keywords: Composite resins; Silicones; Esthetics, Dental.

\title{
Resumen
}

La odontología estética avanza constantemente debido a los procedimentos adhesivos y al desarrollo de materiales restauradores que buscan mejorar las propriedades mecánicas y estéticas para reproducir las características naturales de las estructuras dentales. Este estúdio tiene como objetivo resaltar las ventajas clínicas de su uso, así como su aplicación clínica a través de una revisión de la literatura. Las palabras clave "resinas compuestas", "siliconas" y "estética dental" se utilizaron en las bases de datos PubMed y BVS. Las referencias que trataron los temas relacionados con el tema propuesto fueron elegidas, publicadas entre 1993 
y 2020. Las resinas compuestas actuales permiten restaurar la forma y la función con características de color, translucidez y opacidad similares a las de los dientes naturales, siendo indicadas para la recuperación. La armonía de la sonrisa anterior. El uso de la barrera de silicona para realizar la rehabilitación estética previa facilita el proceso de estratificación y la recuperación estética de la sonrisa, como se puede ver en esta revisión de la literatura.

Palabras clave: Resinas compuestas; Siliconas; Estética dental.

\section{Introdução}

Quando se trata da estética dentária, as pessoas buscam soluções harmoniosas e, para isso, vários fatores devem ser considerados em conjunto, como idade do paciente, tamanho, cor e formato dos dentes e dimensão facial. Os aspectos relativos à cor, morfologia e posicionamento dentário são essenciais para planejar corretamente o tratamento. (Correia, Oliveira \& Silva, 2005).

A recuperação estética do sorriso envolve procedimentos que avaliam o bom senso do profissional e a capacidade de absorção, além de conhecimento e domínio de técnicas restauradoras e de materiais modernos. Para a execução de restaurações com aparência natural é necessário compreender os parâmetros de fluorescência, opalescência, translucidez e cor, de modo a aplicar as diferentes camadas de resina composta presentes em um determinado sistema. Esta aplicação de resina composta em diferentes camadas é denominada de estratificação natural (Higashi, Souza, Liu \& Hirata, 2008).

A utilização de diferentes tipos de resina composta fotopolimerizável para confecção de restaurações classe IV com o auxílio da barreira de silicone, associado à técnicas manuais de confecção da restauração, permite que o cirurgião dentista realize procedimento estéticos com aspecto natural, semelhante ao da estrutura dentária. A técnica de estratificação de compósito é, portanto, a melhor forma de aperfeiçoar a utilização dos materiais adesivos atuais. (Hirata, 2010).

A técnica da barreira de silicone facilita a confecção da estética do sorriso, de forma intrínseca, pois permite que a restauração seja confeccionada a partir da face palatina, seguindo para a face vestibular, utilizando diferentes cores de resina composta, tornando o resultado promissor. Essa barreira auxilia tanto na confecção da restauração, orientando os limites, determinados anteriormente no enceramento diagnóstico, quanto no desgaste necessário para que haja espaço suficiente para o material restaurador preencher o bordo incisal e faces vestibular e palatina (Almeida, Freitas, Barros, Batista \& Soares, 2019). 
Assim, associado aos benefícios descritos na literatura sobre o uso de barreiras de silicone, este estudo tem como objetivo destacar as vantagens do seu uso, bem como sua aplicação clínica.

\section{Metodologia}

As revisões bibliográficas como considera Pereira devem especificar as fontes de consulta, o período de cobertura e os critérios utilizados para as pesquisas. O presente trabalho é uma revisão de literatura, na qual foi realizada uma busca bibliográfica nas bases de dados PubMed e BVS e livros que relatavam os temas propostos de 1993 a 2020 . As palavras-chave utilizadas foram "resinas compostas", "silicones" e "estética dentária".

Os critérios de inclusão foram artigos que abordassem o tema da presente revisão de literatura, sem restrição de idioma, artigos de estudo in vitro, in vivo, relato de caso, revisão de literatura e livros cuja literatura proveria melhores evidências para este trabalho.

Primeiramente, foram selecionados de acordo com os títulos dos trabalhos e posteriormente analisados os resumos. Trabalhos que não apresentaram metodologia detalhada no resumo ou não correspondessem ao tema proposto foram excluídos, dessa forma, 32 artigos foram utilizados.

\section{Revisão de literatura}

\section{Aspectos Gerais referentes a Cor}

O matiz é definido como sendo a família da cor, podendo existir matizes referentes a qualquer uma das cores existentes no espectro de luz visível. Para as resinas compostas, convencionalmente definiu-se que existem quatro matizes: A (marrom), B (amarelo), C (cinza) e D (vermelho), patenteados pela empresa Vita Zahnfabrik (Higashi, Souza, Liu \& Hirata, 2008.).

Clinicamente pode-se dizer que cerca de $80 \%$ dos pacientes possui matiz A e os demais, matiz B e apenas 5\% apresentam o matiz D. Recentemente o matiz C foi removido da escala Vita por não ser considerado um matiz puro. O croma, segunda dimensão da cor, é a saturação de um determinado matiz. Este é identificado nas resinas compostas por uma numeração gradual de 1 a 7, que indica a saturação da resina composta de forma crescente (Costa, 2003). 
O valor pode ser conceituado como a quantidade de preto e branco em um objeto, ou seja, é uma escala de vários tons de cinza. Esta dimensão da cor provoca sensações de profundidade, e é relacionada com a opacidade e translucidez. Quanto menor o valor, mais opaco será o objeto e quanto maior o valor, mais translúcido e acinzentado esse objeto será. O valor é o mais importante das três dimensões citadas para o efeito policromático (Magne \& Holz, 1996). As características ópticas presentes em dentes naturais são determinadas pela interação de luz entre a dentina, o esmalte e a polpa, e incluem vários graus de translucidez e opacidade do esmalte e dentina, fluorescência e opalescência (Feilzer, de Gee \& Davidson, 1993).

Para a seleção de cor é necessário que todas as definições anteriormente citadas estejam bem claras para o cirurgião-dentista. A seleção de cor deve ser feita antes do isolamento absoluto, porque durante o isolamento, os dentes desidratam, resultando em um valor elevado (dentes mais claros e opacos), o que leva a uma seleção de cor incorreta (Vanini, 1996). O mais adequado que a seleção de cor seja feito em luz natural, e quando não é possível como em dias chuvosos, no período noturno ou em consultórios com janelas escuras, a seleção deve ser feita sob fonte de iluminação artificial adequada. O paciente deve estar sentado evitando incidência de luz excessiva perpendicular ao dente, o que pode induzir o dentista à seleção de cores mais claras devido à alta reflexão de luz. Geralmente, o matiz é o mesmo nos três terços do dente, o croma é que geralmente varia, conferindo o policromatismo e o cirurgião-dentista deve estar atento a esse detalhe (Winter, 1993; Fondriest, 2003).

Inicialmente, a cor é selecionada com o auxílio da escala Vita Classical, que usualmente determina primeiro a cor do esmalte que vai por cima da restauração. Deve-se comparar rapidamente a escala de cor com o dente, evitando fixar o olhar na escala, o que acarretará perda da percepção de tons pela fadiga das células cone da retina. Se isto acontecer, deve-se olhar para um objeto azul ou cinza durante a seleção de diferentes cores (Reis, Higashi \& Loguercio, 2009). Após a seleção com a escala, a cor é testada colocando-se um pequeno incremento de resina composta na face incisal do dente e é polimerizada de acordo com as especificações do fabricante (Silva, Reis, Vilela \& Menezes, 2015). O mesmo é feito com todos os terços do dente a ser restaurado, quando necessário. $\mathrm{O}$ pequeno incremento e o dente são então umedecidos com a própria saliva do paciente para simular a aparência de um compósito polido e, só então podemos determinar ser a resina selecionada coincide exatamente com o dente natural (Fahl, Denehy \& Jackson, 1995).

A diferença de espessura de esmalte e dentina ao longo do dente produz diferentes regiões de luminosidade e absorção de luz. Quanto maior a quantidade de dentina maior 
absorção de luz e menor reflexão desta, ficando o dente mais escuro. Onde a espessura do esmalte é maior, ocorre maior reflexão de luz, causando o efeito de maior luminosidade (Mondelli, 2018). Desta forma, as resinas para dentina devem apresentar opacidade suficiente para reproduzir esse efeito de absorção de luz. O esmalte, por sua vez, deve apresentar diferentes níveis de translucidez (Fahl, 1995).

A fluorescência, propriedade onde a substância absorve a energia da luz ultravioleta e emite depois radiação dentro do espectro de luz visível, é um componente importante que deve estar reproduzida nas restaurações e que confere à restauração vitalidade e luminosidade. Os dentes apresentam uma fluorescência predominantemente branca com ligeiro tom azul, sendo a dentina muito mais fluorescente que o esmalte. A opalescência é a propriedade ótica de um material translúcido que lhe dá um aspecto leitoso, com reflexos irisados. Ela se manifesta principalmente na reflexão de luz azul no bordo incisal e no tom alaranjado no colo dos dentes. Esta propriedade permite ao esmalte refletir luz azul e transmitir a tonalidade laranja da dentina. $\mathrm{Na}$ translucidez, há extravasamento disperso da luz, mas também há reflexão dispersa (Behle, 2001; Neto, 2003).

Durante a seleção de cor, o cirurgião-dentista deve estar atento para essas diferenças de absorção e reflexão de luz, optando por uma resina composta com opalescência e fluorescência para a reprodução da dentina e dos mamelos de desenvolvimento e por uma resina composta translúcida e de esmalte para a confecção da região incisal e de esmalte (Choi, Condon \& Ferracane, 2000).

Para que a restauração fique à semelhança dos dentes naturais, a reprodução da espessura do esmalte e da dentina deve ser atingida. Isso permite que a reflexão e a absorção de luz pela restauração seja o mais próximo do dente natural (Calixto, Clavijo, Kabbach \& Andrade, 2008). O cirurgião-dentista não pode esquecer que geralmente as resinas compostas microhíbridas, antes da polimerização apresentam um tom mais claro (alto valor e menor croma) e que depois da polimerização esse tom se torna mais escuro (baixo valor e maior croma), por isso a importância de a cor ser selecionada usando um pequeno incremento de resina, além da escala de cor (Margeas, 2010).

É interessante que o profissional execute uma restauração de diagnóstico (mockup), com as cores selecionadas para auxiliá-lo a avaliar se as cores estão satisfatórias e para mostrar ao paciente o provável resultado a ser obtido. Assim sendo, sem condicionar o esmalte do dente, ele deverá fazer uma restauração com as mesmas resinas e com as mesmas cores previamente selecionadas para o caso, respeitando as espessuras que terão na restauração definitiva (Blum, 2006). 


\section{Proporção Estética Dos Dentes}

A aparência estética de um sorriso é governada pela simetria e proporcionalidade dos dentes (proporção áurea) e pela localização da linha média (Nahsan et al., 2012). Atualmente, o que pode ser usado é uma proporção pré-estabelecida (no caso a proporção áurea de 1,618 para 1,0) entre a largura dos incisivos central e lateral e manter essa proporção constante no posicionamento dos dentes e espaços remanescentes (Wang, Li \& Liu, 2020).

Se a mesma proporção de aparecimento entre a largura do incisivo central e lateral é repetida entre o incisivo lateral e a quantidade do canino que é mostrada, e entre o canino e o primeiro pré-molar, e assim por diante, a largura ou o tamanho do dente serão diferentes, mas estarão relacionados por causa da repetição da mesma proporção (Vieira, 2009).

De acordo com Mondelli (2018), o cirurgião-dentista deve conhecer, além da proporção áurea, as larguras e alturas médias das coroas dos dentes anteriores para que ele possa detectar desarmonias estéticas e alcançar um resultado final em que possa eliminar essas desarmonias.

Dentes mais largos e retangulares transmitem sensação de força e masculinidade, enquanto dentes estreitos e arredondados, sensação de delicadeza e feminilidade. Em contra partida, um estudo realizado por Corbella (2019) e colaboradores com o objetivo de avaliação visual por fotografia frontal se as características morfológicas dentárias são associadas com a determinação do sexo do indivíduo e concluíram que não houve diferença na capacidade de determinar o sexo entre os grupos, sugerindo que a percepção sexual não é fortemente influenciada pelas características dentárias.

A importância do valor numérico do comprimento das coroas clínicas dos dentes anteriores deve ser considerada juntamente com a largura mesiodistal. Não seria possível aplicar corretamente as características geométricas da morfologia dentária sem as informações apropriadas sobre os valores dimensionais médios.

A relação largura e, comprimento da coroa ideal para os: incisivos centrais superiores é de 70 a $80 \%$. Quando o cirurgião-dentista é responsável por restaurar um único elemento dentário, ele pode reproduzir as dimensões e características do dente homólogo. Porém, para Mondelli (2018), quando ele é responsável por uma reabilitação de bateria anterior, ele deve levar em consideração a proporção dos dentes no arco e a proporção estética destes. 


\section{Seleção da Resina Composta}

As restaurações diretas estéticas com resina composta constituem uma excelente opção de tratamento graças à preservação da estrutura dental, uma vez que não há necessidade de confecção de bisel para mascarar a interface dente / resina, técnica rápida e simples (Velo, Coelho, Basting, Amaral \& França, 2016).

A significante evolução das resinas compostas em suas propriedades óticas permite uma infinidade de possibilidades de obtenção de restauração com coloração, translucidez, opacidade e opalescência semelhante ao dente natural (Rodrigues, Argolo \& Cavalcanti, 2014).

A técnica de estratificação das diferentes camadas de resina composta implica em um resultado estético satisfatório não só em termos de cor, mas principalmente na reprodução das propriedades óticas dos dentes naturais, sendo necessário um conhecimento profundo dos materiais que utilizados, bem como da técnica de inserção (Schwarz, Simon, Silva, Ghiggi \& Cericato, 2013).

A crescente busca dos pacientes por restaurações imperceptíveis tem exigido cada dia mais atenção do cirurgião-dentista à técnica de estratificação da resina composta. Heintze \& Rousson (2012) afirmam que o planejamento inicial com modelo de estudo e a etapa restauradora com o uso da matriz palatina facilita a confecção das restaurações e permite que o formato final dos dentes alcançe o desejado.

\section{$\underline{\text { Resina composta microparticulada }}$}

Para, a resina microparticulada, Koottathape, Takahashi, Iwasaki, Kanehira \& Finger (2012) é a resina composta de partículas de sílica coloidal como carga inorgânica que têm aproximadamente $0.04 \mu \mathrm{m}$ de tamanho.

Uma das vantagens desta resina é a obtenção de alto grau de polimento e a manutenção do mesmo, porém devido a pouca quantidade de matriz inorgânica essas resinas apresentam como desvantagem uma baixa resistência mecânica.

Também pela presença de grande quantidade de matriz orgânica ocorre um alto grau de absorção de pigmentos, o que resulta em manchamento, principalmente em margens delgadas . 


\section{$\underline{\text { Resina composta híbrida }}$}

Possui dois tipos de partículas de carga, a sílica coloidal e partículas de vidro contendo metais pesados (os metais pesados oferecem radiopacidade suficiente para detecção radiográfica de cáries secundárias e demais necessidades de diagnóstico).

O tamanho reduzido das partículas de carga, assim como a grande quantidade de micropartículas de sílica coloidal, aumenta a área superficial. As vantagens destas resinas consistiam maior resistência mecânica frente a situações de estresse oclusal com relativo polimento superficial. Sua dificuldade consiste em manter esse polimento (Velo, Coelho, Basting, Amaral \& França, 2016).

\section{$\underline{\text { Resina composta microhíbrida }}$}

As resinas híbridas sofreram modificação e houve uma diminuição no tamanho das partículas maiores para diâmetros em torno de $0,4 \mu \mathrm{m}$, e a resina composta microhíbrida, por ter suas partículas reduzidas acaba possuindo uma maior capacidade de manutenção de polimento. Esta resina é a de escolha para os casos de restaurações classe IV, devido ao seu potencial de polimento e resistência mecânica (Kocaagaoglu, Aslan, Gürbulak, Albayrak, Taşdemir \& Gumus, 2017).

\section{Contração de polimerização e fator de configuração cavitária (Fator C)}

Durante a polimerização das resinas compostas, ocorre a aproximação e a união de suas moléculas, transformando os monômeros em cadeias poliméricas, substituindo espaços de Van der Walls por ligações covalentes (Choi, Condon \& Ferracane, 2000; Davidson \& Feilzer, 1997).

A quantidade de material que se contrai quando esta passa de estado gel para estado sólido define a contração de polimerização. As resinas compostas contraem entre $1 \%$ a $3 \%$ do seu volume. Se a tensão de contração for maior que a força de união entre resina / sistema adesivo e o dente, a interface pode ser rompida dando origem a uma fenda. Esta interface defeituosa pode ser a principal causa de falha desta restauração, por predisposição à infiltração e consequente descoloração marginal, sensibilidade pós-operatória e cáries 
recorrentes, diminuindo a longevidade da restauração (Silva, Bandeira, Cunha, Magalhães, Lopes \& Freitas, 2017).

O Fator C é descrito como sendo a razão entre a área de superfícies aderidas e a área de superfícies livres, determinando desta maneira a relação entre a forma do preparo cavitário e a capacidade de alívio das tensões provenientes da contração de polimerização. Este alívio depende da capacidade de escoamento dos materiais, ou seja, da sua deformação elástica e seu escoamento para as superfícies livres, relaxando as tensões da contração e possibilitando uma melhor união adesiva (Davidson \& Feilzer, 1997).

Levando-se em consideração o Fator C, o escoamento das resinas compostas é muito limitado em cavidades de classe I e V, sendo que possuem Fator $\mathrm{C}=5$, ou seja, cinco paredes aderidas (vestibular, lingual, mesial, distal e pulpar) e apenas uma parede livre (oclusal). Em cavidades de classe IV o Fator $\mathrm{C}$ é mais favorável, $\mathrm{C}=2,0 / 5,0$. Em cavidades de classe II e III o Fator C fica entre $\mathrm{C}=1,0 / 2,0$. A partir destes estudos foram desenvolvidas várias técnicas que objetivam o controle do Fator $\mathrm{C}$ e dos efeitos resultantes da contração de polimerização (Feilzer, de Gee \& Davidson, 1993).

Uma das técnicas desenvolvidas foi à incremental, que consiste na polimerização de pequenos incrementos de resina composta, de no máximo 2,0 $\mathrm{mm}$, que diminui a ocorrência de fator $\mathrm{C}$ porque a adesão da resina se restringe a poucas paredes, deixando uma área livre para o escoamento e alívio das tensões, além da menor quantidade de material que irá se contrair (Davidson \& Feilzer, 1997).

\section{Guia de silicone}

Uma guia de feita com material de impressão a base de silicone pode ser confeccionada a partir da própria restauração existente, quando o paciente queixa-se da alteração da cor do dente restaurado e está satisfeito com o formato; ou então a partir de um enceramento diagnóstico no modelo de estudo, quando o paciente quer uma reabilitação do sorriso ou apresenta alguma fratura (Fonseca, Kasuya, Favarão, Honorato \& Filho, 2013).

A barreira confeccionada a partir de um enceramento diagnóstico, além de orientar os limites da restauração, a barreira orienta o desgaste dental necessário para a confecção da restauração. Para as duas formas de confecção, a barreira não pode abranger a face vestibular dos dentes, apenas a face palatina e a metade da face incisal. Essa barreira vai servir como apoio para a confecção da face palatina do dente que será restaurado (Mishra, Yeluri, Garg \& Ralla, 2015). 
O dente a ser restaurado deve passar primeiramente por profilaxia e depois pelo condicionamento ácido com ácido fosfórico $37 \%$ por 15 a 30 segundos, depois deve ser lavado com jato de água e ar por mais 30 segundos. O cirurgião-dentista deve secar o dente com uma bolinha de algodão úmido para manter a dentina úmida, esse procedimento impede o colapso das fibrilas de colágeno da dentina, possibilitando a infiltração dos monômeros do adesivo nos espaços interfibrilares. O sistema adesivo é então aplicado no dente, de acordo com as especificações técnicas do fabricante, formando a camada híbrida. O cirurgião dentista não pode se esquecer de proteger o dente adjacente do condicionamento ácido e da aplicação do adesivo, bem como mantê-lo protegido durante todo o procedimento restaurador (Baratieri, 2001).

Assim que o dente já estiver com o adesivo, a barreira de silicone é posicionada para a confecção da parede palatina do dente. A cor selecionada para a última camada de esmalte é usada para a confecção da parede palatina do dente. Essa parede é confeccionada pela técnica incremental, e a barreira só é removida quando a face palatina estiver totalmente confeccionada (Mondelli, 2008).

Com os limites da restauração já definidos, a barreira pode ser removida e o processo de estratificação se inicia, aplicando pequenos incrementos de resina composta da cor de dentina com característica opaca e opalescente, selecionada anteriormente, à semelhança dos mamelos de desenvolvimento. O profissional deve estar atento ao dente adjacente e observar a forma destes, para reproduzi-los no dente a ser restaurado. Na borda incisal, em pacientes jovens, uma resina opaca branca pode ser usada para a confecção do halo opaco incisal. Cada pequeno incremento é polimerizado pelo tempo determinado pelo fabricante da resina composta (Baratieri, 2001).

Esse procedimento é repetido até que a restauração chegue à resina composta de esmalte, que será a última camada. É interessante o uso de pincéis durante todo o procedimento restaurador da face vestibular para a reprodução da textura do dente. A textura é tão importante quanto a seleção de cor para o efeito natural da restauração, uma vez que esta interfere na reflexão da luz (Prado et al., 2012).

\section{Acabamento e polimento}

O final do tratamento restaurador se dá com o acabamento e polimento da restauração. O primeiro passo é a remoção dos excessos vestibulares e palatinos usando-se 
pontas diamantadas para acabamento de resina composta de granulação fina e extra fina; e dos excessos proximais, usando-se uma tira de lixa para resina composta (Baratieri, 2001).

O ajuste oclusal da restauração anterior deve ser feito com o paciente em repouso e os movimentos mandibulares devem ser consultados para que não haja interferências durante a desoclusão do paciente. Para este ajuste, o cirurgião-dentista também deve utilizar pontas diamantadas de granulação fina (Silva, Reis, Vilela \& Menezes, 2015).

Na sequência, o cirurgião-dentista deve delimitar as áreas de reflexão de luz com um lápis, comparando o tamanho dessa área com o auxílio de um compasso de ponta seca, entre o dente restaurado e o dente natural homólogo. Se a simetria não estiver presente, um disco abrasivo de óxido de alumínio pode ser usado para o acabamento da restauração. Estas linhas de brilho interferem no comprimento e na largura do dente. A diminuição da área plana entre as linhas promove menor reflexão de luz, gerando a ilusão óptica de um dente mais estreito. Ao contrário disso, ao aumentar esta área, haverá maior reflexão de luz e, consequentemente, um aspecto de dente mais largo será observado (Prado et al., 2012).

Durante o processo de acabamento, o cirurgião-dentista pode realizar com o auxílio de pontas diamantadas de granulação fina uma evidenciação na textura do dente restaurado, principalmente dos sulcos vestibulares. Estes procedimentos de acabamento devem ser realizados em baixa rotação através de contra-ângulo (Silva, Reis, Vilela \& Menezes, 2015).

Após a remoção dos excessos, a remoção das irregularidades das superfícies planas pode ser realizada com borrachas siliconizadas de diferentes granulações, da granulação mais grossa para a granulação mais fina. Sempre que o cirurgião-dentista mudar a granulação da ponta, ele deve lavar bem o dente restaurado para que não fique resíduos da outra ponta na restauração. Para a finalização da restauração, um disco de feltro é aplicado sobre a restauração com uma pasta diamantada para polimento de resina composta (Prado et al., 2012).

\section{Considerações Finais}

As restaurações estéticas se tornam rotina constante do consultório odontológico. Para tanto, habilidade técnica do uso de artifícios, como o uso da guia de silicone para a realização das reabilitações estéticas, facilitam o processo de estratificação com resina composta. 


\section{Referências}

Almeida VSC, Freitas MPC, Barros VPV, Batista MRO, Soares, GM. (2019). Restauração com resina composta anterior pela técnica da barreira de silicone - caso clínico. Rev. Bras. Odontol., 76:(Supl.2), 118.

Baratieri, LN. (2001). Restaurações adesivas diretas em dentes anteriores fraturados. In: Odontologia restauradora: fundamentos e possibilidades. São Paulo: Santos.

Behle, C. (2001). Shade selection techniques: Part three - principles for stratification. Pract Periodont Aesthet Dent, 13(9), 717-20.

Blum, I. (2006). Composite restorations in anterior teeth: fundamentals and possibilities. $\mathrm{Br}$ Dent J, 201, 313.

Calixto, LR, Clavijo, V, Kabbach, W, Andrade, MF. (2008). Harmonização do sorriso com resina composta direta. $R$ Dental Press Estét, Maringá, 6(1), 18-28.

Choi, KK, Condon, JR \& Ferracane, JL. (2000). The effects of adhesive thickness on polymerization contraction stress of composite. J Dent Res, 79(3), 812-17.

Corbella, S, Alberti, A, Muzzarelli, M, Zotti, B, Taschieri, S \& Francetti, L. (2019). Anatomical factors associated with gender recognizability: A study on intraoral standardized photographs. J Investig Clin Dent, 10(4).

Correia, A, Oliveira, MA \& Silva, MJ. (2005). Conceitos de Estratificação nas restaurações de dentes anteriores com resinas compostas. Revista Portuguesa de Estomatologia, Medicina dentária e Cirurgia Maxilofacial, 46(3), 171-78.

Costa, FLM. (2003). Trabalho de conclusão apresentado ao Curso de Especialização em Dentistica. Florianopólis.

Davidson, CL, Feilzer, A. (1997). Polymerization shrinkage and polymerization shrinkage stress in polymer-based restoratives. $J$ Dent, 25(6), 35-40. 
Fahl, N, Denehy, G \& Jackson, R. (1995). Protocol for predictable restoration of anterior teeth with composite resins. Pract Periodonto Aesthet Dent, 7(7), 13-21.

Fahl, N. (1995). Predictable aesthetic reconstruction of fracture anterior teeth with composite resins: a case report. Pract Periodont Aesthet Dent, 8(1), 17-31.

Feilzer, AJ, de Gee AJ, Davidson CL. (1993). Setting stresses in composites for two different curing modes. Dent Mater, 9(1), 2-5.

Fondriest, J. (2003). Shade matching in restorative dentistry: the science and strategies. Int J Periodontics Restorative Dent, 23(5), 467-479.

Fonseca, RB, Kasuya, AVB, Favarão, IN, Honorato, ISS, Filho, LCA. (2013). Técnica de Estratificação e Texturização Superficial de Resinas Compostas em Dentes Anteriores - Seis Meses de Acompanhamento. Clínica - International Journal of Brazilian Dentistry, 9(3), 324332.

Heintze, SD \& Rousson, V. (2012). Clinical effectiveness on direct class II restorations: a metaanalysus. $J$ Adhes Dent, 14(5), 407-431.

Higashi, C, Souza, CM, Liu, J \& Hirata, R. (2008). Resina composta para dentes anteriores. In: Fonseca AS. Odontologia Estética: a arte da perfeição. São Paulo: Artes Médicas.

Hirata, R. (2010). Tips, Dicas Em Odontologia Estética. São Paulo: Artes médicas.

Kocaagaoglu, H, Aslan, T, Gürbulak, A, Albayrak, H, Taşdemir, Z, Gumus, H. (2017). Efficacy of polishing kits on the surface roughness and color stability of different composite resins. Niger J Clin Pract, 20(5), 557-565.

Koottathape, N, Takahashi, H, Iwasaki, N, Kanehira, M, Finger, WJ. (2012). Two and threebody wear of composite resins. Dent Mater, 28(12), 1261-70. 
Magne, P \& Holz, J. (1996). Stratification of composite restorations: Systematic and durable replication of natural esthetics. Pract Periodontics Aesthet Dent, 8(1), 61-68.

Mondelli, J. (2018). Estética e Cosmética em Clínica Integrada Restauradora, São Paulo: Quintessence.

Margeas, RC. (2010). Keys to success in creating esthetic class IV restorations. $J$ Esthet Restor Dent, 22(1), 66-71.

Mishra, A, Yeluri, R, Garg, N \& Rallan, M. (2015). Putty silicone as a guide in the restorative managment of primary double tooth: A case report. Annals of Dental Specialty, 3(1), 21-23.

Nahsan, FPS, Mondelli, RFL, Franco, EB, Naufel, FS, Ueda, JK, Schmitt, VL \& Baseggio, W. (2012). Clinical strategies for esthetic excellence in anterior tooth restorations: understanding color and composite resin selection. J Appl Oral Sci, 20(2), 151-6.

Neto, CL. (2003). Estética do sorriso - Arte e Ciência. São Paulo: Santos.

Pereira, AS et al. (2018). Metodologia da pesquisa científica. [e-book]. Santa Maria. Ed. UAB/NTE/UFSM. Acesso em: 6 maio 2020. Disponível em: https://repositorio.ufsm.br/bitstream/handle/1/15824/Lic_Computacao_MetodologiaPesquisa-Cientifica.pdf?sequence $=1$.

Prado, M, Gomes, BPFA, Telles, EL, Araújo, MCP \& Gusman, HC. (2012). Fratura coronorradicular: uma abordagem multidisciplinar. R. Odontol. UNESP, 41(5), 360-364.

Reis, A, Higashi, C \& Loguercio, AD. (2009). Re-anatomization of aterior eroded teeth by stratification with direct composite resin. J Esthet Restor Dent, 21(5), 304-317.

Rodrigue, SDR, Argolo, S, Cavalcanti, AN. (2014). Reanatomização dental com resina composta - Relato de Caso. Revista Bahiana de Odontologia, 5(3), 182-192.

Schwarz, V, Simon, LS, Silva, SA, Ghiggi, PC \& Cericato, GO. (2013). Fechamento de diastema com resina composta: Relato de caso. J Oral Invest, 2(1), 26-31. 
Silva, FP, Reis, GR, Vilela, ALR \& Menezes, MS. (2015). Reabilitação estética de dente fraturado - relato de caso. Full Dent. Sci., 6(22), 249-255.

Silva, JDS, Bandeira, ACFM, Cunha, ML, Magalhães, APR, Lopes, LG \& Freitas, GC. (2017). Forças de contração de polimerização em resinas compostas constituídas com novas matrizes orgânicas e fotoativadas por diferentes técnicas. Rev Odontol Bras Central, 26(79), $21-25$.

Vanini, L. (1996). Light and color in anterior composite restorations. Pract Periodontics Aesthet Dent, 8(7), 673-682.

Velo, MMAC, Coelho, LVBF, Basting, RT, Amaral, FLB \& França, FMG. (2016). Longevity of restorations in direct composite resin: literature review. $R G O, 64:(3), 320-326$.

Vieira, D. (2009). Análise do Sorriso, São Paulo: Santos.

Wang, P, Li, DJ, Liu, JZ. (2020). Analysis of the relationship among maxillary anterior teeth width, anterior arch perimeter and anterior segment depth. Beijing Da Хие Xие Bao Yi Хиe Ban, 52(1), 124-128.

Winter, R. (1993). Visualizing the natural dentition. J Esthet Dent, 5(3), 102-117.

\section{Porcentagem de contribuição de cada autor no manuscrito}

Guereth Alexsanderson Oliveira Carvalho - 40\%

Rubiana Romão de Almeida - 15\%

João Victor Frazão Câmara - 15\%

Amanda de Oliveira Pinto Ribeiro - 15\%

Josué Junior Araujo Pierote - 15\% 